\title{
Relación entre tamaño de partículas en heces de equinos y calidad nutricional del forraje consumido
}

\author{
Pellegrini, $A G^{1,2}{ }^{1,}$ Topayan, $M V^{1,2}$; Borlandelli, MS. ${ }^{1,2}$; Abbiati, NN. ${ }^{1,2}$; Paz, $S^{1,2}$ \\ 1. Facultad de Ciencias Agrarias, Universidad de Lomas de Zamora, Ruta $4 \mathrm{~km}$ 2, Lomas de \\ Zamora (1836) Buenos Aires. \\ 2. Instituto de Investigación sobre Producción Agropecuaria, Ambiente y Salud
}

Correo electrónico: apellegrini@supeq.com.ar.

\section{RESUMEN}

En la producción y salud de caballos, la nutrición es crítica; los equinos son herbívoros no rumiantes y conocer la calidad de los forrajes que pastorean, resulta primordial para la valoración nutricional. Nos propusimos evaluar la proporción de tamaños de partículas de heces frescas de equinos que pastoreaban distintos tipos de forrajes. Utilizamos el instrumento comercial Nasco Digestion Analyzer para lavar las muestras de heces frescas y pasarlas por una estructura de 3 tamices de acero inoxidable con diámetro de poro decreciente, siendo: A:> 4,8 mm; B: 4,8-3,2 mm; C: 3,2-1,6 mm y D:<1,6 mm, el remanente que escapa. Se obtuvieron 31 muestras de heces frescas de yeguas adultas de raza Sangre Pura de Carrera. Los tratamientos fueron: 1.Dieta Pp (n:9) pastura consociada; 2.Dieta Ss (n:8) verdeo de soja; 3.Dieta Mn (n:7) verdeo de maíz y 4.Dieta Aa (n:7) verdeo de avena. Las muestras de forrajes fueron analizadas para materia seca (MSf), digestibilidad in vitro (DIG), fibra en detergente neutro (FDN) y fibra en detergente ácido (FDA). Nuestros resultados evidenciaron que las proporciones acumuladas por tamaño de partículas del material de heces frescas, son diferentes según el tipo de dieta ( $\mathrm{p}:<0,05)$, para tamaño de partículas mayor a 4,8 mm (tamiz A), siendo los valores para cada dieta: Pp: 47\%, Ss: 27\%, Mn: 13\% y Aa: 2\%. También se encontraron relaciones entre parámetros de calidad nutricional de los forrajes pastoreados y tamaños de partículas en las heces frescas con niveles estadísticos significativos $(p<0,05)$, a saber: DIG:A, r: -0,51 (p: 0,0037); DIG:D, r: 0,57 (p: 0,0008); FDN:A, r: 
0,51 (p: 0,0034); FDN:D, r: -0,56 (p: 0,0011); FDA:A, r: 0,59 (p: 0,0005); FDA:A, r: 0,63 (p: 0,0001). Posiblemente haga falta un mayor número de evaluaciones de la interacción forraje/heces, con este método, para poder definir un modelo descriptivo al respecto

Palabras claves: Equinos, nutrición, crecimiento, digestibilidad.

\section{Relationship between particle size in equine feces and nutritional quality of forage consumed}

\section{ABSTRACT}

In horses production and health, nutrition is critical; equines are non-ruminant herbivores and knowing the quality of forages that graze, is essential for nutritional assessment. Thus, we set out to evaluate the proportion of particle sizes of fresh feces of horses that graze different types of forages. We used the commercial instrument Nasco Digestion Analyzer to wash the samples of fresh feces and pass them through a structure of 3 stainless steel sieves with decreasing pore diameter, being: A:> $4.8 \mathrm{~mm}$; B: $4.8-3.2 \mathrm{~mm}$; C: $3.2-1.6 \mathrm{~mm}$ and $\mathrm{D}:<1.6 \mathrm{~mm}$, the remnant that escapes. 31 samples of fresh feces were obtained from adult Race Purebred Horse mares. The treatments were: 1 . Diet Pp (n: 9) consociated pasture; 2. Diet Ss (n: 8) soy summer greengrass; 3. Diet Mn (n: 7) corn summer greengrass and 4.Diet Aa (n: 7) oatmeal summer greengrass. The forage samples were analyzed for dry matter (MSF), in vitro digestibility (DIG), fiber in neutral detergent (NDF) and fiber in acid detergent (ADF). Our results show that the proportions accumulated by particle size of fresh stool material are different according to the type of diet (p: <0.05), for particle size greater than $4.8 \mathrm{~mm}$ (sieve A), being the values for each diet: Pp: 47\%, Ss: $27 \%$, Mn: $13 \%$ and Aa: $2 \%$. We also found relationships between parameters of nutritional quality of grazed forages and particle sizes in fresh feces with significant statistical levels $(\mathrm{p}<0.05)$, namely: DIG: A, $r$ : -0.51 (p: 0) , 0037); DIG: D, r: 0.57 (p: 0.0008); FDN: A, r: 0.51 (p: 0.0034); FDN: D, r: -0.56 (p: 0.0011); FDA: A, r: 0.59 (p: 0.0005); FDA: A, r: 0.63 (p: 0.0001). Possibly, more evaluations about the forage / feces interaction with this method are needed to be able to define a descriptive model in this regard.

Key words: Equines, nutrition, growth, digestibility.

Fecha de recepción artículo original: 02-09-2019

Fecha de aceptación para su publicación: 10-11-2019 


\section{Introducción}

En Argentina, el modelo de producción equina tradicionalmente pastoril representa una importante ventaja competitiva a nivel mundial, tanto por sus reducidos costos como por un manejo semi-extensivo valorado en el mercado ${ }^{(1)}$. En la producción y salud de los caballos, la nutrición juega un papel crítico ${ }^{(2)}$. La calidad de la dieta ha sido identificada como un regulador clave de la situación nutricional, condición corporal, tasa de supervivencia, éxito reproductivo y en última instancia, de la dinámica de poblaciones de herbívoros mamíferos ${ }^{(3)}$. Desde el punto de vista estrictamente alimenticio, los equinos son herbívoros no-rumiantes ${ }^{(4)}$ por lo que el conocimiento de la composición y calidad de los forrajes que pastorean, resultan primordiales para la valoración de su nutrición ${ }^{(5)}$.

Las múltiples pasturas y verdeos implantados que componen la cadena forrajera sufren variaciones en cuanto a su contenido de nutrientes a lo largo del año debido básicamente a las condiciones edafo-climáticas y a sus respectivos avances en la madurez fenológica ${ }^{(6,7)}$. Resulta prácticamente imposible disponer de los parámetros de calidad por composición química de los forrajes pastoreados en cada momento.

Asimismo, y coincidiendo con lo que expresa Sales et al. ${ }^{\left({ }^{8}\right)}$, los métodos de análisis de la digestibilidad in vivo tienden a necesitar muchas horas de consumo, mucha mano de obra, con costos muy altos, y resultan impracticables. Proponemos usar el instrumento comercial Nasco Digestion Analyzer para observar posibles cambios en heces frescas como una guía clave a considerar en la evaluación de la alimentación (9), siendo ésta, una técnica de bajo costo y fácil implementación, como método complementario para la valoración nutricional de la calidad del forraje ofrecido a los equinos.

\section{Materiales y métodos}

\section{RECLUTAMIENTO DE ANIMALES Y TOMA DE MUESTRAS}

Se trabajó con 31 yeguas adultas de raza Sangre Pura de Carrera (de rango etario entre 6-12 años de edad), todas en óptimo estado de salud de acuerdo a lo establecido por el médico veterinario y con condición corporal entre 5 a 7 puntos en la escala de 1 a 9 según Henneke et al ${ }^{(10)}$. Los animales formaban parte de los planteles de tres haras ubicados en la localidad de San Antonio de Areco (Pcia de Buenos Aires) y cumplían con el programa sanitario de cada lugar. Se eligió 
esta zona, perteneciente a la región Pampeana, dado que la misma se establece como una de las de mayor importancia en la industria hípica nacional.

Para la realización de este trabajo se tomaron muestras de heces frescas (n:31) en yeguas que estaban pastoreando cada tipo de forraje por más de 14 días, asegurando así por un lado, un período de adaptación adecuado y por el otro, superar el tiempo de retención máximo de la digesta en el tracto gastrointestinal (TGI) en los equinos ${ }^{(11)}$, para asegurar que el material encontrado en las heces tenga relación directa con el consumido.

Las heces frescas fueron tomadas directamente desde recto o del suelo inmediatamente post defecación asegurando que no haya contaminación con tierra u otros restos. Las mismas fueron guardadas en bolsas individuales y rotuladas con fecha, registro particular y establecimiento. Fueron conservadas en heladera hasta su procesamiento para evitar pérdida de agua.

Se clasificaron los tipos de Dietas (tratamientos) en función a los distintos forrajes pastoreados con su respectiva característica botánica y de estacionalidad de producción, logrando así armar grupos funcionales diferentes (Forraje: Heces) por cada lote evaluado. Quedando así 4 tratamientos o tipos de Dieta como se expresa en la siguiente tabla:

\begin{tabular}{|c|c|c|c|}
\hline Dieta & n & Período & Descripción \\
\hline Dieta Pp & 9 & Primavera 2016 & $\begin{array}{c}\text { Pastura a base de rye grass (Lolium Multiflorum), } \\
\text { trébol blanco (Trifolium repens) y cebadilla criolla } \\
\text { (Bromus unioloides). }\end{array}$ \\
\hline Dieta Ss & 8 & Verano 2017 & $\begin{array}{c}\text { Verdeo de soja (Glycine max) en estado fenológico } \\
\text { R5. }\end{array}$ \\
\hline Dieta Mn & 7 & Verano 2017 & $\begin{array}{c}\text { Verdeo de maíz (Zea mayz) en estado fenológico } \\
\text { R2 - R4. }\end{array}$ \\
\hline Dieta Aa & 7 & Invierno 2017 & Verdeo de Avena (Avena sativa) en estado vegetati- \\
vo temprano.
\end{tabular}

\section{ANÁLISIS DE CALIDAD NUTRICIONAL DE LAS DIETAS}

Se tomaron muestras representativas de cada forraje de los respectivos potreros dónde pastoreaban los animales. Los lotes estaban parejos en disponibilidad y estado fenológico, con lo cual se dispuso la extracción de 5 sub-muestras en forma aleatoria para hacer cada muestra. La altura de corte fue realizada de acuerdo al patrón de consumo de los animales en cada caso. Las muestras de forraje fueron guardadas en bolsas de nylon rotuladas con identificación de la 
especie/mezcla forrajera, fecha y establecimiento. Fueron preservadas en heladera hasta su procesamiento.

Para la valoración de la calidad nutricional de los forrajes se analizaron en el Laboratorio de la Facultad de Ciencias Agrarias, UNLZ., los parámetros: materia seca (MSf), digestibilidad in vitro (DIG), fibra en detergente neutro (FDN) y fibra en detergente ácido (FDA).

Para determinación de MSf las muestras fueron secadas en estufa a $70^{\circ} \mathrm{C}$ hasta peso constante. El análisis de DIG se efectuó por medio del sistema ANKOM (Daisy II, ANKOM Corp., Fairtport, NY, EEUU). Las muestras secas fueron molidas a $1 \mathrm{~mm}$, colocándose 0,25 gr de material en bolsas ANKOM llevadas a digestión en solución de pepsina con ácido clorhídrico $(\mathrm{HCl}) 0,1 \mathrm{~N} \mathrm{a} 40^{\circ} \mathrm{C}$ por $24 \mathrm{hs}$. Luego las muestras fueron lavadas con agua destilada a punto de ebullición hasta $\mathrm{pH}$ neutro y posteriormente fueron colocadas en solución de celulasa por $30 \mathrm{hs}$ a $40^{\circ} \mathrm{C}$. Para la determinación de FDN y FDA fueron colocados $0,50 \mathrm{gr}$ de material seco y molido a $1 \mathrm{~mm}$ en bolsas ANKOM identificadas individualmente. Las mismas fueron llenadas con $2000 \mathrm{ml}$ con detergente neutro y detergente ácido, correspondientemente, a temperatura ambiente. Las muestras fueron dejadas en el medio por 60 minutos (según métodos descriptos por Goering y Van Soest ${ }^{(12)}$, modificadas por Van Soest y Robertson ${ }^{(13)}$ y y adaptados en el Laboratorio NIRS. Facultad de Ciencias Agrarias, Universidad Nacional de Lomas de Zamora).

\section{EVALUACIÓN DEL TAMAÑO DE PARTÍCULAS DE LAS HECES}

Las muestras de heces frescas fueron evaluadas a través del uso del instrumento comercial Nasco Digestion Analyzer (www.enasco.com) para la determinación de tamaño de partículas.

a

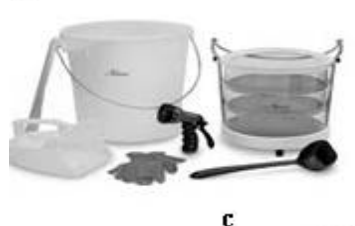

b

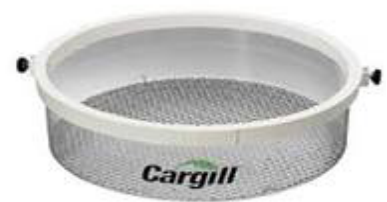

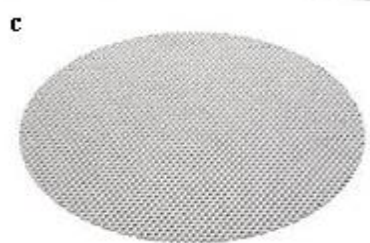

Imagen 1. a: Equipo comercial Nasco Digestion Analyzer completo con todos los utensilios; b: se muestra con mayor detalle la bandeja donde queda el material retenido y c: tamiz de acero cada uno con su respectivo tamaño de poro. 
El mismo consiste en una estructura que sostiene 3 tamices de acero inoxidable que disminuyen el diámetro de poro desde el superior hacia el inferior (denominamos con letras cada estrato o tamiz): (A), (B) y (C), respectivamente. Se consideró como variable residuo (D) a la proporción que escapó a los tres estratos.

\begin{tabular}{|c|c|c|c|c|}
\hline Estrato o Tamiz & A & B & C & D \\
\hline Rango de tamaño de partícula en $\mathrm{mm}$ & $>\mathbf{4 , 8}$ & $\mathbf{4 , 8 - 3 , 2}$ & $\mathbf{3 , 2 - 1 , 6}$ & $<\mathbf{1 , 6}$ \\
\hline
\end{tabular}

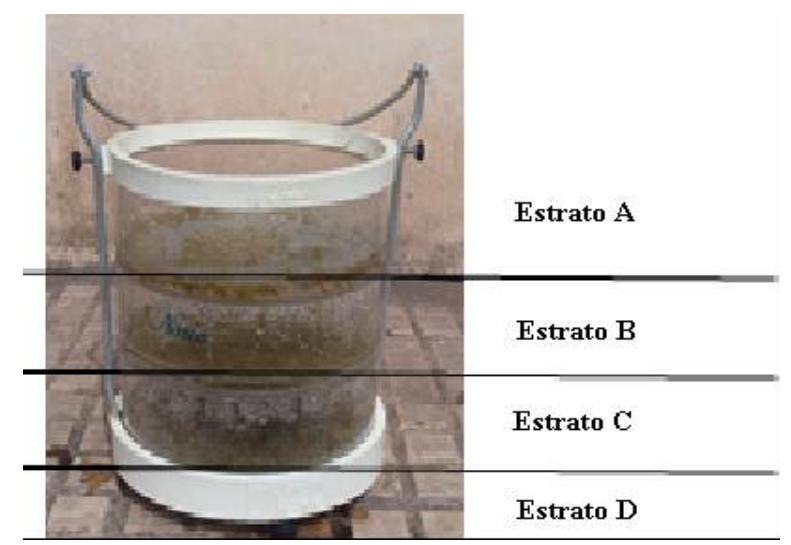

Imagen 2. Estructura que sostiene las tres bandejas, cada una con un tamiz de distinto tamaño de poro.

Para esta determinación las muestras de heces frescas en rango de $110 \mathrm{gr}$ - 160gr fueron sometidas a lavado con aspersor durante $4 \mathrm{mi}$ nutos. Se registró el peso para las fracciones retenidas en cada estrato, para luego calcular la proporción relativa de cada uno de ellos. Se determinó el valor promedio de cada estrato para cada dieta.

\section{ANÁLISIS DE DATOS Y ESTADÍSTICA}

El procesamiento y análisis de datos incluye estudio de relación entre las variables de calidad nutricional del forraje y tamaño de partículas expresado como proporción retenida en cada estrato (análisis de correlación). Se efectuaron análisis de varianza y comparación de medias por medio de Test de Tukey. Previamente se estudiaron los supuestos, y en caso que no se cumpliera el supuesto de homogeneidad de varianzas, en el análisis de varianza se empleó un modelo mixto con varianzas heterogéneas para el error. Se usaron los software estadísticos Infostat ${ }^{(14)}$ y SAS ${ }^{(15)}$ (y se trabajó con un nivel de significación del 0,05). 


\section{Resultados}

\section{VALORACIÓN NUTRICIONAL DE LAS DIETAS}

En la tabla 1 se describen las propiedades de los forrajes a través de los valores DIG (\%), FDA (\%), FDN (\%) y MSf (\%) para cada una de las dietas.

\begin{tabular}{|c|c|c|c|c|c|c|}
\hline Dieta & Forraje & $\begin{array}{c}\text { Estado } \\
\text { Fenológico }\end{array}$ & DIG (\%) & FDA (\%) & FDN (\%) & MSf (\%) \\
\hline Pp & Pastura & Veg. Tardío & 66,0 & 32,4 & 55,3 & 19,2 \\
\hline Ss & Soja & R5 & 81,5 & 26,1 & 44,5 & 28,4 \\
\hline Mm & Maíz & R2 - R4 & 63,6 & 32,2 & 59,6 & 16,6 \\
\hline Aa & Avena & Veg. Temprano & 87,3 & 23,4 & 34,3 & 15,1 \\
\hline
\end{tabular}

Tabla 1. Valores de composición y calidad de los forrajes para cada una de las dietas. Dónde: $\mathrm{Pp}=$ pastura, $\mathrm{Ss}=$ soja, $\mathrm{Mm}=$ maíz, $\mathrm{Aa}=\mathrm{Avena}, \mathrm{DIG}=$ digestibilidad In vitro, FDN= fibra en detergente neutro, FDA= fibra en detergente ácido, MSf= materia seca del forraje.

En líneas generales y como era de esperar, a menor porcentaje de fibra (expresada como FDN y FDA) mayor la digestibilidad de los forrajes ${ }^{(2,4)}$. El valor más elevado de DIG se registró para la Avena (87.3\%) coincidiendo con un estado fenológico vegetativo muy temprano al momento de corte ${ }^{(6)}$. En forma decreciente siguen la Soja, Maíz y registrándose el valor más bajo en la Pastura (66.0\%). Todos los valores de DIG son mayores a los rangos medios encontrados en las tablas nutricionales de referencia ${ }^{(2,4)}$. La concentración de materia seca (\%MSf), fue mayor para la Soja valor $(28,4 \%)$ seguido, en forma decreciente por la Pastura, Maíz y Avena.

\section{TAMAÑO DE PARTÍCULAS EN HECES FRESCAS POR TIPO DE DIETA}

En forma descriptiva, vemos en el grafico 1 los promedios de las proporciones retenidas del material de heces frescas de cada estrato para cada tipo de dieta, evidenciando a priori diferencias detectables. Partículas de tamaño mayor a 4,8 $\mathrm{mm}$ (A) y menor a 1,6 $\mathrm{mm}$ (D) son más relevantes en tal distinción. Los estratos $\mathrm{B}$ y $\mathrm{C}$ fueron siempre minoritarios para los forrajes evaluados. 


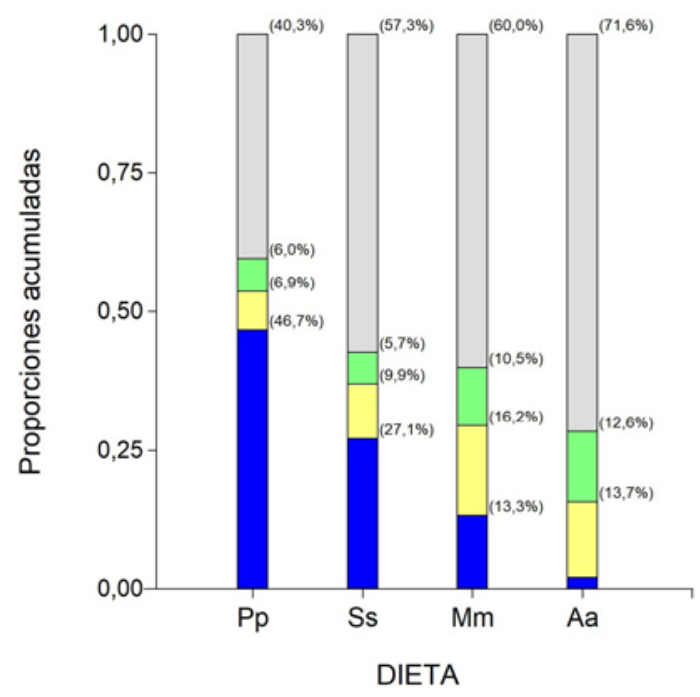

Gráfico 1. Proporciones acumuladas de los estratos A (azul), B (amarillo), C (verde) y D (gris) para todas las dietas. Dónde: $\mathrm{Pp}=$ pastura, $\mathrm{Ss}=\mathrm{soja}, \mathrm{Mm}=$ maíz, $\mathrm{Aa}=$ avena.

Las medidas resumen para las variables A, B, C y D para todas las dietas, se muestran en la tabla 2. Observamos que dentro de cada grupo y dependiendo del tamiz evaluado los coeficientes de variación son muy variables. De todas maneras, es importante remarcar que para el tamiz o estrato con mayor proporción de material retenido los \% $\mathrm{CV}$ son menores: siendo para Dieta Aa - estrato D $(0,72) \% \mathrm{CV}: 7,81$; Dieta $\mathrm{Mn}$ - estrato D $(0,60) \% \mathrm{CV}: 5,24$; Dieta Pp - estrato A $(0,47) \% \mathrm{CV}: 14,15$ y Dieta Ss - estrato D $(0,57) \% C V: 11,55$.

\begin{tabular}{|c|c|c|c|c|c|c|c|c|}
\hline DIETA & Estrato & $\mathrm{n}$ & Media & D.E. & E.E. & CV\% & Mín & Máx \\
\hline Aa & A & 7 & 0,02 & 0,01 & 0,00 & $\mathbf{5 6 , 4 0}$ & 0,01 & 0,04 \\
\hline Aa & $\mathbf{B}$ & 7 & $\mathbf{0 , 1 4}$ & 0,05 & 0,02 & $\mathbf{3 3 , 7 5}$ & 0,08 & 0,19 \\
\hline Aa & $\mathbf{C}$ & 7 & $\mathbf{0 , 1 3}$ & 0,04 & 0,01 & $\mathbf{2 7 , 9 0}$ & 0,06 & 0,16 \\
\hline Aa & $\mathbf{D}$ & 7 & $\mathbf{0 , 7 2}$ & 0,06 & 0,02 & $\mathbf{7 , 8 1}$ & 0,63 & 0,80 \\
\hline Mm & $\mathbf{A}$ & 7 & $\mathbf{0 , 1 3}$ & 0,02 & 0,01 & $\mathbf{1 2 , 6 2}$ & 0,12 & 0,17 \\
\hline Mm & $\mathbf{B}$ & 7 & $\mathbf{0 , 1 6}$ & 0,04 & 0,01 & $\mathbf{2 3 , 8 6}$ & 0,11 & 0,22 \\
\hline Mm & $\mathbf{C}$ & 7 & $\mathbf{0 , 1 0}$ & 0,01 & 0,00 & $\mathbf{1 1 , 8 0}$ & 0,09 & 0,12 \\
\hline Mm & $\mathbf{D}$ & 7 & $\mathbf{0 , 6 0}$ & 0,03 & 0,01 & $\mathbf{5 , 2 4}$ & 0,54 & 0,63 \\
\hline Pp & $\mathbf{A}$ & 9 & $\mathbf{0 , 4 7}$ & 0,07 & 0,02 & $\mathbf{1 4 , 1 5}$ & 0,36 & 0,56 \\
\hline Pp & $\mathbf{B}$ & 9 & $\mathbf{0 , 0 7}$ & 0,07 & 0,02 & $\mathbf{9 8 , 8 6}$ & 0,01 & 0,20 \\
\hline
\end{tabular}




\begin{tabular}{|l|l|l|l|l|l|l|l|l|}
\hline Pp & C & 9 & $\mathbf{0 , 0 6}$ & 0,03 & 0,01 & $\mathbf{4 7 , 3 7}$ & 0,02 & 0,11 \\
\hline Pp & D & 9 & $\mathbf{0 , 4 0}$ & 0,12 & 0,04 & $\mathbf{2 9 , 0 3}$ & 0,24 & 0,59 \\
\hline Ss & A & 8 & $\mathbf{0 , 2 7}$ & 0,06 & 0,02 & $\mathbf{2 1 , 3 1}$ & 0,20 & 0,37 \\
\hline Ss & B & 8 & $\mathbf{0 , 1 0}$ & 0,06 & 0,02 & $\mathbf{5 6 , 7 0}$ & 0,05 & 0,21 \\
\hline Ss & C & 8 & $\mathbf{0 , 0 6}$ & 0,01 & 0,00 & $\mathbf{1 6 , 0 3}$ & 0,04 & 0,07 \\
\hline Ss & D & 8 & $\mathbf{0 , 5 7}$ & 0,07 & 0,02 & $\mathbf{1 1 , 5 5}$ & 0,47 & 0,66 \\
\hline
\end{tabular}

Tabla 2. Valores promedio (media), desvío estándar (D. E), error estándar (E. E), coeficiente de variación (C. V), mínimo (mín) y máximo (máx) para el material retenido en cada estrato. Dónde: $\mathrm{Pp}=$ pastura, $\mathrm{Ss}=$ soja, $\mathrm{Mm}=$ maíz, $\mathrm{Aa}=$ Avena, $\mathrm{A}=$ partículas de heces fresca con tamaño mayor a $4,8 \mathrm{~mm}, \mathrm{~B}=$ partículas de heces frescas con tamaño entre 4,8 - 3,2 mm, C= partículas de heces frescas con tamaño entre $3,2-1,6 \mathrm{~mm}, \mathrm{D}=$ partículas de heces frescas con tamaño menor a $1,6 \mathrm{~mm}$.

Dado que para las variables B y D se halló homogeneidad de varianzas (PValue $>0,05$ ), se puede observar que sí existen diferencias significativas entre las medias para las dos variables. Las mismas fueron analizadas mediante la prueba de comparación de medias Test de Tukey, con un nivel de confianza del $95 \%$ para la variable B (PValue $>0,05$ ) y un nivel de confianza del $99 \%$ para la variable D (PValue $>0,01)$. Se encontró que las medias correspondientes a las dietas para tamaño de partículas entre $3,2-4,8 \mathrm{~mm}$ se dividen en dos grupos, sin observarse diferencias intragrupo: Pp, Ss y Aa y Ss, Aa y Mm; pero sí hallándose diferencias entre las dietas Pp y Mm (grafico 2). Para tamaño de partículas 1,6-3,2 mm, las medias de las dietas Ss y Mm no difieren entre sí pero si con las medias correspondientes a Pp y Aa.
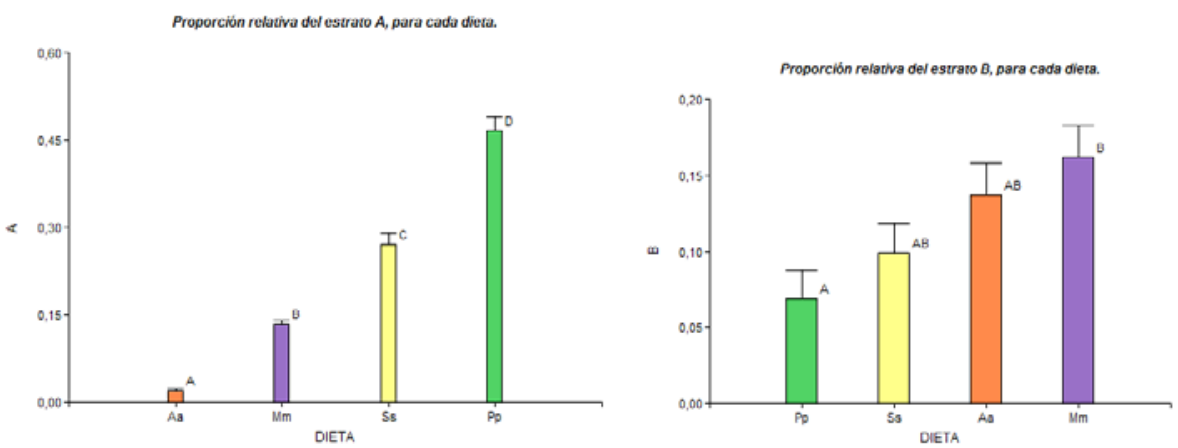

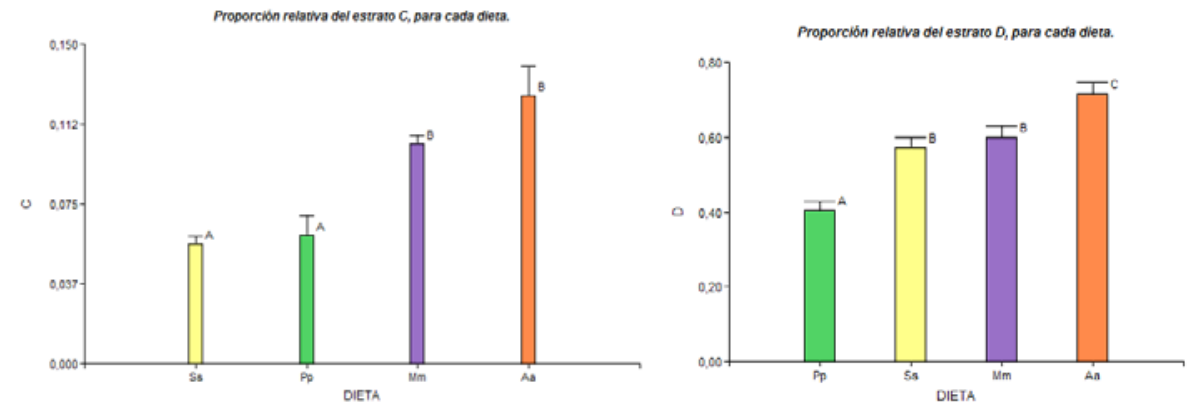

Gráfico 2. Proporción promedio de los estratos A, B, C y D relativa al total para todas las dietas. Letras diferentes representan diferencias significativas entre medias. Dónde: $\mathrm{Pp}=$ pastura, $\mathrm{Ss}=$ soja, $\mathrm{Mm}=$ maíz, $\mathrm{Aa}=$ avena.

Por otra parte, dado el incumplimiento del supuesto de homogeneidad de varianza para las variables A y C, se llevó a cabo el análisis estadístico con un modelo mixto que contempló la heterogeneidad de las varianzas por medio del software estadístico SAS (2018). Para ambas variables se encontraron diferencias significativas entre medias correspondientes a cada una de las dietas $(\mathrm{P}<0,05)$. Para tamaño de partículas mayor a $4,8 \mathrm{~mm}$ se hallaron diferencias significativas entre las medias correspondientes a las cuatro dietas (Gráfico 2).

Finalmente, para la variable $C$, se encontró la existencia de dos grupos con evidencia de diferencias: las medias correspondientes a las dietas Ss y Pp no difieren entre sí como tampoco lo hacen $\mathrm{Mm}$ y Aa (Gráfico 2), a pesar que el análisis de valoración nutricional no presenta similitudes entre las dietas (Tabla 1). Por lo tanto, el rango de particulas entre 1,6-3,2 $\mathrm{mm}$ no es posible diferenciar entre tipo y calidad de dieta consumida.

\section{RELACIÓN ENTRE TAMAÑO DE PARTÍCULAS EN HECES FRESCAS Y CALIDAD DE LA DIETA}

La Tabla №3 muestra los coeficientes de correlación de Pearson entre los parámetros de valoración de calidad nutricional de los forrajes y el tamaño de partículas en las heces frescas representado por la proporción retenida durante el lavado en cada estrato. Para la variable MSf no se evidencian relaciones significativas, excepto con la variable correspondiente a partículas de tamaño entre 3,2-1,6 mm (C). En este caso en particular, se registró una correlación moderada y negativa $(r=-0,61)$ manifestando que el incremento en una de las variables implica la reducción de la otra. Para el resto de las variables (DIG, FDN y FDA) se hallaron correlaciones moderadas y de significancia aparente con las variables tamaño de 
partículas mayor a 4,8 $\mathrm{mm}$ y menor a 1,6 $\mathrm{mm}$. Sin embargo se observan dos patrones de comportamiento. Por un lado, se manifiesta una correlación inversa para el par DIG-A $(\mathrm{r}=-0,51)$ y directa para DIG-D $(\mathrm{r}=0,57)$; por otro lado, FDA y FDN exponen el comportamiento opuesto. Los pares FDN-A y FDA-A expresan correlación positiva ( $\mathrm{r}=0,51$ y $\mathrm{r}=0,59$, respectivamente) mientras que los pares FDN-D y FDA-D se correlacionan en forma inversa ( $r=-0,53$ y $r=-0,63$, respectivamente).

\begin{tabular}{|c|c|c|c|c|}
\hline \multicolumn{5}{|c|}{ Correlación de Pearson } \\
\hline MSf & A & 31 & 0,38 & 0,0362 \\
\hline MSf & B & 31 & $-0,27$ & 0,1486 \\
\hline MSf & C & 31 & $-0,61$ & 0,0003 \\
\hline MSf & D & 31 & $-0,20$ & 0,2799 \\
\hline DIG & A & 31 & $-0,51$ & 0,0037 \\
\hline DIG & B & 31 & 0,05 & 0,7776 \\
\hline DIG & C & 31 & 0,22 & 0,2285 \\
\hline DIG & D & 31 & 0,57 & 0,0008 \\
\hline FDN & A & 31 & 0,51 & 0,0034 \\
\hline FDN & B & 31 & $-0,05$ & 0,8053 \\
\hline FDN & C & 31 & $-0,29$ & 0,1115 \\
\hline FDN & D & 31 & $-0,56$ & 0,0011 \\
\hline FDA & A & 31 & 0,59 & 0,0005 \\
\hline FDA & B & 31 & $-0,11$ & 0,5503 \\
\hline FDA & C & 31 & $-0,29$ & 0,1157 \\
\hline FDA & D & 31 & $-0,63$ & 0,0001 \\
\hline
\end{tabular}

Tabla 3. Coeficientes de correlación de Pearson entre las variables de composición del forraje y tamaño de partículas de las heces. Dónde: $\mathrm{MSf}=$ materia seca del forraje, $\mathrm{A}=$ partículas de heces frescas con tamaño mayor $4,8 \mathrm{~mm}, \mathrm{~B}=$ partículas de heces frescas con tamaño entre 4,8-3,2 mm; $C=$ partículas de heces frescas con tamaño entre 3,2 - 1,6 mm, D= partículas de heces frescas con tamaño menor a 1,6 mm, DIG= digestibilidad In vitro, FDN= fibra en detergente neutro, FDA= fibra en detergente ácido.

\section{Discusión}

\section{TAMAÑO DE PARTÍCULAS EN HECES DE EQUINOS}

El presente trabajo pudo discriminar el tamaño de partículas en heces frescas de equinos, utilizando el instrumento comercial (Nasco Digestion Analyzer), como método práctico y económico cuando 
pastorean distintos tipos de forrajes. En un estudio anterior con el mismo aparato ${ }^{(9)}$ lavaron las heces de vacas lecheras con agua tibia y utilizaron un tiempo de 30 segundos. En nuestro caso, no dispusimos de un sistema para calentar el agua y tuvimos que incrementar el tiempo de lavado con agua fría a 4 minutos. Más que la temperatura del agua, consideramos que el factor primordial que explica el tiempo extra necesario para lavar las heces frescas es la diferencia entre las heces de los bovinos y equinos. Las partículas fecales de los rumiantes son más pequeñas que la de los equinos ${ }^{(16)}$, en promedio $0,2 \mathrm{~mm}$ en ovinos y 0,4 mm en bovinos ${ }^{(17)}$. Estos investigadores también determinaron un tamaño medio de partículas fecales en equinos de 1,8 \pm $0,8 \mathrm{~mm}$; usaron mayor cantidad de forrajes y mallas de apertura de $8 \mathrm{~mm}, 6,3 \mathrm{~mm}, 4 \mathrm{~mm}, 2,5 \mathrm{~mm}, 1,25 \mathrm{~mm}, 0,8 \mathrm{~mm}, 0,4 \mathrm{~mm}$ y $0,16 \mathrm{~mm}$. En nuestro estudio no se realizó la valoración del tamaño medio de partículas en heces.

Se presume que el tamaño de partículas en las heces es representativo del tamaño de partículas de la digesta después del proceso masticatorio. Los equinos dedican muchas horas del día a pastorear y tienen un proceso masticatorio muy intenso, entre 3000 a 3500 movimientos para comer un kg de heno ${ }^{(4)}$. También ocurre una separación entre dos fases de la digesta en el tracto posterior del equino debido a retención selectiva de grandes partículas. El movimiento coordinado de los músculos circulares y longitudinales sobre la porción de TGI colon ventral izquierdo-flexura pélvica-colon dorsal izquierdo causan una separación física de partículas menos digeridas y más groseras - más de $1 \mathrm{~cm}$ aproximadamente ${ }^{(11)}$. Este proceso de retención diferencial, aumenta el tiempo de fermentación de la porción fibrosa de los forrajes.

De acuerdo a nuestros resultados, la proporción de material fecal retenido en los tamices varía con el tipo de forraje consumido. La tasa de reducción de las partículas, la densidad de la digesta y características del flujo son factores importantes que pueden afectar las tasa de pasaje y así el nivel de consumo y la digestibilidad (17). En nuestro estudio, hubo diferencias significativas $(\mathrm{P}<0,05)$ entre las dietas para tamaño de partículas mayor a 4,8 mm (A). Y si bien hubo estrecha relación entre estratos A y D (coeficiente de correlación Pearson r: -0,870) no hubo diferencias significativas para partículas menores a 1,6 mm (D). En el análisis de varianza de esta variable para tipo de dieta, no se encontraron diferencias entre las dietas de soja (Ss) y maíz (Mn). Esto podría deberse a efectos indirectos sobre la digestión por el posible aporte de grano que tenía la planta de maíz (estado fenológico R2-R4) interactuando su almidón con la fibra durante el proceso de fermentación en el tracto posterior. 
Si bien en nuestro trabajo no se estudió como variable de análisis las características dentarias de las yeguas, varios grupos de investigadores han estudiado sus efectos sobre la digestión en equinos. Trabajos de corrección dentaria ${ }^{(18)}$, evaluación de movimientos rostro-caudal de la mandíbula ${ }^{(19)}$ como medición del ángulo oclusal de los molares ${ }^{(20)}$. Siendo los resultados contradictorios, aparentemente el grado de anormalidad dentaria fue distinto entre los estudios y por lo tanto no puede asumirse con precisión la influencia de las características dentarias o proceso masticatorio sobre el tamaño de partículas encontrado en las heces.

\section{RELACIÓN DE LAS HECES CON LA CALIDAD DE LA DIETA}

Así como también lo expresa Bastianelli et al. ${ }^{(21)}$, nuestro trabajo se sustenta en que las heces son el producto final del proceso digestivo y por la tanto contienen información del alimento consumido y del paso de la digesta por el tracto gastrointestinal. Estudios previos han podido relacionar la composición química en heces con la valoración nutricional del alimento consumido. Tolleson and Schafer ${ }^{(22)}$ indican que la tecnología de análisis NIRS en heces puede ser usado para monitorear la calidad de la dieta. Así pueden monitorear el estado nutricional de rodeos de vacunos en importantes regiones áridas o semiáridas. En parte, coinciden con Kidane et al. ${ }^{(23)}$ quienes realizan análisis de heces de burros por NIRS. En su investigación, han notado que el contenido de proteína cruda de la dieta fue predicho con niveles aceptables de exactitud, mientras que para digestibilidad fue menos acertada. Un trabajo realizado con heces de equinos para predecir la calidad de las dietas ${ }^{(24)}$ sugiere que los índices fecales pueden ser muy útiles en la predicción de la digestibilidad, obteniendo la mayor precisión por regresión múltiple con valores de proteína y fibra en las heces.

En nuestra investigación, se demuestran las relaciones, medidas a través del análisis de correlación de Pearson, entre las variables de calidad del forraje (DIG, FDN y FDA) y el tamaño de partículas de las heces frescas (Estratos A, B, C y D). Las relaciones más significantes $(p<0,05)$ fueron siempre con los tamaños de partículas mayor a 4,8 $\mathrm{mm}(\mathrm{A})$ y menor a 1,6 mm (D). Siendo el patrón de comportamiento, cuanto mayor la digestibilidad de la dieta menos material queda en el estrato $\mathrm{A}$ y más en D; y contraria pero lógicamente, cuanto más fibra expresado tanto en FDN como en FDA tienen los forrajes más cantidad de material queda retenido en el estrato A y menos en D. Nuestros resultados son coincidente con el trabajo de Grenet et al. ${ }^{(17)}$ donde los coeficientes de correlación muestran que los \% de partículas fecales mayor a 0,16 mm 
incrementan cuando la digestibilidad de la materia orgánica y la fibra cruda de los forrajes disminuyen. Para la variable materia seca de las heces, nosotros no encontramos ningún tipo de diferencia significativa entre las dietas ni tampoco ningún nivel de relación importante con la calidad de los forrajes. Hutjens ${ }^{(9)}$ si tuvo tendencia estadísticamente significativa entre el contenido de FDN del alimento y la materia seca en las heces de vacas lecheras. Posiblemente, en nuestro caso hubiese sido necesario evaluar mayor cantidad de pares Heces/Forrajes para generar más oportunidades en tales relaciones. Por otro lado y a fin de evitar una alteración en el patrón de consumo, las yeguas muestreadas estuvieron permanentemente en la parcela, evitando así cambios bruscos en la tasa de ingesta que lleve a implicaciones para la salud del sistema gastrointestinal y afectar parámetros en la digestibilidad o de las heces ${ }^{(25)}$.

Evaluar los parámetros físicos de las heces a fin de relacionarlo con la calidad de los distintos forrajes disponibles para su consumo sigue siendo de especial interés para la valoración nutricional de programas de alimentación en equinos. Kohnke ${ }^{(26)}$ (1992) ha descripto un sistema de evaluación de las heces frescas para monitoreo el proceso de digestión en equinos tomando en cuenta la consistencia, el olor y color. Aunque parece práctico carece de objetividad en su análisis. En nuestro caso, a través del separador de partículas, pudimos inicialmente diferenciar por tipo de forraje consumido, a través de las proporciones del material encontrado en las heces frescas (indigestible). El valor nutritivo de las pasturas dependerá del contenido de nutrientes que aporten (27), de las disponibilidad de los mismos para el animal, de la eficiencia con que se absorben y son utilizados y también de los posibles efectos sobre el consumo ${ }^{(28)}$. La digestibilidad de los diferentes nutrientes puede ser afectada por varios factores, los cuales pueden ser propios del animal, del alimento o también del ambiente ${ }^{(29)}$.

\section{Conclusión}

Este trabajo muestra por primera vez en nuestro medio, la interacción entre el tipo de forraje de la dieta y el tamaño de partículas en las heces equinas. En primera instancia, los forrajes se reducen a partículas muy pequeñas dentro del proceso digestivo de los equinos, y esta disminución depende del tipo y calidad de los forrajes consumidos.

El uso del instrumento Nasco Digestion Analyzer para caracterizar las heces frescas de los equinos a través de la medición de la proporción de partículas de diferente tamaño, podría ser un método práctico, sencillo y económico para interpretar la calidad nutricional del forraje 
pastoreado. Seguramente, esto será de mayor utilidad en los sistemas extensivos de producción equina.

Consideramos que harían falta un mayor número de evaluaciones de la interacción forraje/heces con este método para poder definir un modelo descriptivo y posteriormente predictivo al respecto. 


\section{Bibliografía}

1. Paz S; Pellegrini AG.. Construyendo el Sistema de Producción con Énfasis en la Nutrición Equina. Criollos. En: Anuario de Asociación Criadores de Caballos,2008, 97:174-177.

2. NRC. 2007. Nutrient Requeriment of Horses. 6th Edition. Washington, D.C. U.S.

3. Gil-Jiménez, E; Villamuelas, M; Serrano, E; Delibes, M; Fernández, N; Fecal Nitrogen Concentration as a Nutritional Quality Indicator for European Rabbit Ecological Studie, Journal PLoS ONE, 2015, Vol 10 (4).

4. INRA. 2015. Equine nutrition, INRA nutrient requirements, recommended allowances and feed tables. Ed. Wageningen Academic Publishers. The Netherlannds, 696p.

5. Cymbaluk, NF. Comparison of forage digestion by cattle and horses, Journal Animal Science, 1990, 70: 601-610.

6. 6. Cangiano, CA.1997. Producción Animal en Pastoreo. INTA. Ed. La Barrosa, Argentina, $145 p$.

7. Darlington JM; Hershberger TV. Effect of Forage Maturity on Digestibility, Intake and Nutritive Value of Alfalfa, Timothy and Orchardgrass by Equine. Journal Animal Science, 1968, 27: 1572-1576.

8. Sales J; Homolka P; Koukolová V. Prediction of Energy Digestibility of Hays in Horses. Journal of Equine Veterinary Science, 2013, xxx 1-7.

9. Hutjens MF. Finding Feeding Bottlenecks on Dairy Farms. En Four State Dairy Nutricion and Managment. Conference, Iowa 2016 Junio 15- 16. Universidad Estatal de Iowa, 2016, p. 92-97.

10. Henneke DR, Potter GD, Kreider JL and Yeates BF. Relationship Between Condition Score, Physical Measurements and Body Fat Percentage in Mares. Equine Veterinary Journal,1983, 15 (4):371 - 372.

11. Van Weyenberg S, Sales J and Janssens GPJ.. Passage rate of digesta through the equine gastrointestinal tract: A review. Livestock Science 2006, 99: 3-12.

12. Goering HK and Van Soest PJ.. Forage fiber analyses, Apparatus, Reagents, Procedures, and Some Applications. Agriculture Handbook No. 379. Agricultural Research Service,1970, Washington, D.C. 24p.

13. Van Soest PJ and Robertson JB. 1980. Systems of analysis for evaluating fibrous feeds. En: Standardization of Analytical Methodology in Feeds. Pigden, W J; Balch, C C; Graham, M, International Research Development Center, Ottawa, Canada.pp. 49-60.

14. Di Rienzo JA; Casanoves F; Balzarini MG; Gonzalez L; Tablada M; Robledo CW. InfoStat versión 2011. Grupo InfoStat, FCA, Universidad Nacional de Córdoba, Argentina.

15. SAS. 2018. Institute Inc. SAS ${ }^{\circledR}$ 9.4. Cary, NC: SAS Institute Inc., USA.

16. Uden P; Van Soest PJ.. The determination of digesta particle size in some herbivores. Animal Feed Science and Technology, 1982, 7: 35-44.

17. Grenet E; Martin-Rosset W; Chenost M.. Compared size and structure of plant particles in the horse and the sheep feces. Journal Animal Science, 1984, 64:345-346. 
18. Zwirglmaier, S; Remler HP; Senckenberg E; Fritz J; Stelzer P; E. Kienzle.. Effect of dental correction on voluntary hay intake, apparent digestibility of feed and faecal particle size in horse. Journal of Animal Physiology and Animal Nutrition, 2011, 1-8.

19. Carmalt JL; Allen AL.. Effect of rostrocaudal mobility of the mandible on feed digestibility and fecal particle size in horses. Journal of the American Veterinary Medical Association, 2006, 229: 1275-1278.

20. Carmalt JL; Cymbaluk NF; Townsend HGG.. Effect of premolar and molar occlusal angle on feed digestibility, water balance, and fecal particle size in horses. Journal of the American Veterinary Medical Association 2005, 227:110-113.

21. Bastianelli D; Bonnal L; Jaguelin-Peyraud Y; Noblet J.. Predicting feed digestibility from NIRS analysis of pig faeces. Journal Animal, 2015, 9(5): 781-786.

22. Tolleson DR; Schafer DW.. Application of fecal near-infrared spectroscopy and nutritional balance software to monitor diet quality and body condition in beef cows grazing Arizona rangeland. Journal Animal Science, 2014, 92: 349-358.

23. Kidane NF; Stuth JW; Tolleson DR.. Predicting Diet Quality of Donkeys via Fecal-NIRS Calibrations. Rangeland Ecology \& Management, 2008, 61 (2): 232-239.

24. Mésochina, P; Martin-Rosset W; Peyraud JL: Duncan D; Micol D; Boulot S.. Prediction of the digestibility of the diet of horses: evaluation of faecal índices. Grass and Forage Science, 1998, 53: 189-196.

25. Glunk EC; Pratt-Phillips SE; Siciliano PD. Effect of Restricted Pasture Access on Pasture Dry Matter Intake Rate, and Fecal pH in Horses. Journal of Equine Veterinary Science, 2012, xxx:1-6.

26. Kohnke J. 1992. Feeding and Nutrition. Ed. Birubi Pacific, Australia. 197 p.

27. Pellegrini AG y Paz S. El valor nutritivo del forraje para el equino. La Especie Equina; 2016, 15(55): 44-48

28. Hoskin S0; Gee EK. Feeding value of pastures for horses. New Zealand Veterinary Journal, 2004, 52 (6): 332-341.

29. Topayan MV, Pellegrini AG y Paz S. Pruebas de Digestibilidad en Equinos. La Especie Equina.2017; 16(58): 36-40. 
